\title{
Cuidado Parental à Criança com Paralisia Cerebral: uma Revisão Sistemática da LiTERATURA ${ }^{1}$ \\ Parental Care for Children With Cerebral Palsy: a Systematic Literature REVIEW
}

\author{
Tatiana $\mathrm{AFONSO}^{2}$ \\ Maely Ferreira Holanda RAMOS ${ }^{3}$ \\ Irlana Lessa FRANÇA ${ }^{4}$ \\ Fernando Augusto Ramos PONTES 5 \\ Simone Souza da Costa e SILVA ${ }^{6}$
}

\begin{abstract}
RESUMO: o objetivo da presente revisão foi refletir sobre estudos empíricos acerca da percepção dos pais sobre os cuidados dirigidos ao filho com paralisia cerebral. A busca eletrônica se deu nas bases de dados Psynet APA e utilizou protocolo adaptado a partir das diretrizes da Cochrane Collaboration. A amostra inicial foi composta por 279 estudos, a partir da qual foram realizados dois testes de relevância: o teste de relevância I - aplicado aos resumos dos artigos por um pesquisador e o teste de relevância II- aplicado por dois juízes externos que realizaram a leitura dos artigos na íntegra. Vinte e dois artigos foram selecionados. Os objetivos relatados foram submetidos ao Nvivo 10 software para análise de cluster por decodificaçáo e aplicação de análise de conteúdo. Tais análises revelaram cinco grandes categorias analíticas: reabilitação (serviços centrados na família, percepção dos pais e qualidade de vida das mães durante a reabilitaçáo); experiência nos cuidados diários (dificuldades das mães, a experiência dos pais, sobrecarga e tempo de cuidado); apoio (sofrimento dos pais, apoio do parceiro, estresse, necessidades dos pais, percepção do suporte recebido, problemas comportamentais da criança e serviço de cuidado formal); satisfaçáo dos pais (práticas de serviços centrados na família e a experiência dos pais durante os procedimentos cirúrgicos experimentados pelas crianças) e experiência na alimentação. Concluiu-se que a satisfação dos pais em relação ao apoio recebido e aos serviços especializados se mostrou baixa.
\end{abstract}

PALAVRAS-CHAVE: Educação Especial. Paralisia Cerebral. Cuidado à Criança.

\begin{abstract}
The present systematic review aimed to reflect on empirical studies of parents' perceptions about the care directed to children with cerebral palsy. The electronic search was made on Psynet APA database and used an adapted protocol from Cochrane Collaboration guidelines. The initial sample consisted of 279 studies. Two relevance tests were conducted: test of relevance I - applied by one researcher on the abstracts, and test of relevance II- applied by two external judges that read the articles in full. Twenty-two articles were selected. The objectives of the selected articles underwent Nvivo 10 software for decoding cluster (attribute value) and applying content analysis. The analysis resulted in 5 major analytical categories: rehabilitation (portrayed issues such as family-centered service - FCS, perception of parents and quality of life of mothers during rehabilitation); experience in daily care (highlighting issues as difficulties of mothers of children with CP, parental experience, burden and care time); support (issues that described suffering of parents, spousal support, parental stress, parents' needs, perception of received support, child behavior problems and formal service care); parental satisfaction (FCS practices and the parental experience during surgical
\end{abstract}

\footnotetext{
${ }^{1}$ http://dx.doi.org/10.1590/S1413-65382216000300011

Financiamento: Capes através de bolsa de doutorado

${ }^{2}$ Doutoranda pelo Programa de Pós-Graduação Teoria e Pesquisa do Comportamento, Universidade Federal do Pará, Belém, PA, Brasil. afonso_tatiana@hotmail.com

${ }^{3}$ Doutora e Pós-doutoranda Programa de Pós-Graduação Teoria e Pesquisa do Comportamento, Universidade Federal do Pará, Belém, PA, Brasil.maelyramos@hotmail.com

${ }^{4}$ Vinculada ao Programa de Pós-Graduação Teoria e Pesquisa do Comportamento, Universidade Federal do Pará, Belém, PA, Brasil. Tel. (91) 988436780. irlana.franca@hotmail.com

${ }^{5}$ Professor vinculado ao Programa de Pós-Graduação Teoria e Pesquisa do Comportamento, Universidade Federal do Pará, Belém, PA, Brasil. farp1304@gmail.com

${ }^{6}$ Professora vinculada Programa de Pós-Graduação Teoria e Pesquisa do Comportamento, Universidade Federal do Pará, Belém, PA, Brasil.symon.ufpa@gmail.com
} 
procedures experienced by children) and experiences with food. It is concluded that parents were dissatisfied with the provision of services in rehabilitation, and care practices underscored the emotional wear and tear which involved mainly mothers facing reported difficulties, which in most reports were without support and resources.

KEYWORDS: Special Education. Cerebral Palsy. Child Care.

\section{INTRODUÇÃo}

O cuidado parental se dá a partir da forte conexão entre pais e filhos, sendo constituído por um conjunto de açóes contínuas, influenciadas pelas teias de apoios disponíveis e pelo contexto em que se processa (VIEIRA et al., 2009). Cuidar de uma criança exige dos pais a construção de uma série de aprendizados frente às demandas infantis em que participam fatores de ordem econômica, social, contextual, familiar, relacional e psicológica, podendo ser compreendido, portanto, a partir de diferentes conjunturas e correlaçốes (CURY; BRANDÁO, 2011; CUNNINGHAM, 2008).

Os estudos direcionados ao cuidado parental ganham destaque na literatura quando envolvem circunstâncias crônicas de saúde ou nas deficiências, como é o caso da paralisia cerebral $(\mathrm{PC})$, devido ao impacto gerado frente às dificuldades experimentadas durante o processo de reabilitação. A paralisia cerebral impacta o desenvolvimento da criança de diferentes modos, podendo gerar além dos déficits motores, dificuldades de aprendizagem, distúrbios sensoriais, da fala, entre outras deficiências e/ou problemas de saúde (GERALIS, 2007). Frente a esse diagnóstico, os pais apresentam preocupaçôes com os danos causados e demonstram dúvidas e anseios sobre tratamentos adequados, suporte social e orientaçóes de profissionais especializados (CURY; BRANDÁO, 2011; BELSKY; KELLY, 1994).

Nesse sentido, estudos recentes indicam novas perspectivas em relaçáo ao desenvolvimento da criança com PC e o processo de inclusão social de sua família. Essa tendência vem se fortalecendo em diversas áreas da saúde, reforçando a importância dos cuidados parentais assim como o perfil e a saúde dos cuidadores (CURY; BRANDÁO, 2011; CUNNINGHAM, 2008; GERALIS, 2007).

A família que passa pela experiência do diagnóstico da paralisia cerebral pode experimentar emoçóes e sentimentos conflituosos no confronto com uma realidade que na maioria dos casos é desconhecida e repleta de desafios (GERALIS, 2007). A partir do diagnóstico, a família terá que viver um longo processo de adaptação em que novos hábitos e rotinas são gerados com vistas a atender as necessidades do novo membro, podendo surgir, dessa forma, dificuldades no relacionamento entre pais e crianças (CURY; BRANDÃO, 2011; GERALIS, 2007). Em função desta adaptação, as relações familiares, assim como a vida profissional e social dos pais acabam sendo reformuladas.

O processo de adaptação das famílias diante da deficiência tem fomentado a investigação de fatores psicossociais mediadores do cuidado às crianças com PC. Dentre esses, o estresse (ZHU et al., 2006; HATZMANN et al., 2009; WHITING, 2012), a sobrecarga percebida (FLORIAN; FINDLER, 2001; LOPES et al., 2012), as características da criança (BERGE; PATTERSON; RUETER, 2006; THURSTON et al., 2011), as redes de suporte social (PATRICK; HAY DEN, 
1999; HATZMANN et al., 2009; THURSTON et al., 2011) e as características do cuidador (LIN, 2000; HORTON; WALLANDER, 2001; BRITNER et al., 2003).

Segundo pesquisas recentes, o estresse se mostra como o fator psicossocial mais recorrente, uma vez que prejudica a capacidade dos pais de cuidar de um filho com deficiência. Pais estressados percebem de modo negativo o cuidado e apresentam sensação de sobrecarga uma vez que precisam de mais tempo para cuidar de seus filhos (FLORIAN; FINDLER, 2001; CUNNINGHAM, 2009; LOPES et al., 2012). Além disso, criar um filho com PC acaba expondo as mães, principais cuidadoras, a demandas físicas, psicológicas e sociais que prejudicam sua saúde ao longo dos anos (FLORIAN; FINDLER, 2001) influenciando de forma negativa a adaptação entre pais e crianças com PC.

Tendo em vista tais pressupostos, a presente revisão teve como intuito organizar as principais contribuiçóes da literatura na área da psicologia no sentido de promover a reflexão acerca dos estudos sobre famílias de crianças com paralisia cerebral, sistematizando os dados a partir da categorização dos objetivos apresentados pelas pesquisas selecionadas. A realização do levantamento bibliográfico com essa temática visa auxiliar educadores e profissionais que lidam com famílias de crianças com PC a visualizar a área, assim como planejarem suas açóes.

\section{Método}

Este estudo apresenta uma perspectiva descritiva e exploratória sobre os trabalhos desenvolvidos acerca de pesquisas empíricas na área da psicologia que retratam o cuidado parental à criança com PC. Para tanto, utilizou-se um protocolo constituído por etapas, adaptado das orientaçôes da Colaboração Cochrane (Medicina Baseada em Evidências) para a realização de revisões sistemáticas, a saber: 1) formulação da pergunta/problema; 2) localização e seleção dos estudos; 3) avaliação crítica dos estudos; 4) localização das informações investigadas; 5) análise e apresentação dos dados (CASTRO et al., 2002; CORDEIRO et al.,2007).

Ressalta-se que o método Cochrane foi adaptado na presente revisão, uma vez que fora desenvolvido para ser utilizado em estudos clínicos e sobre intervençóes. Embora tenha sido validado visando pesquisas de natureza diferente, pertencentes a outras áreas do conhecimento, no presente estudo, mostrou-se como uma ferramenta adequada na orientação das etapas de uma boa revisão, pautando-se na experiência positiva de pesquisas que utilizaram essa adaptação nas áreas ligadas à psicologia e educação (ANDRADE DA SILVA; OTTA, 2014).

\subsection{Primeira etapa: FormulaÇáo da PERGunta}

A formulação da pergunta central da revisão sistemática é uma importante etapa, pois nela é possível definir a direção da investigação. Para tanto, utilizou-se a técnica PVO, onde $\mathrm{P}$ refere-se à situação problema, participantes ou contexto; $\mathrm{V}$ compreende as variáveis do estudo; $\mathrm{O}$ aplica-se ao desfecho ou resultado esperado.

O PVO é uma adaptação do modelo PICO visando pesquisas que tratam de temáticas relacionadas à psicologia ou áreas afins. $\mathrm{O}$ modelo PICO foi planejado para atender estudos de caráter clínico, de intervenção, onde P refere-se a participantes, I à intervenção, C a controle 
e $\mathrm{O}$ a resultados (ANDRADE DA SILVA; OTTA, 2014). Essas técnicas permitem organizar os elementos de uma pesquisa para estruturar as perguntas.

Desta forma, com a técnica PVO construiu-se a seguinte questão: como os pais percebem o cuidado ao seu filho (a) com paralisia cerebral? Assim obteve-se a seguinte composição: P = pais de crianças com paralisia cerebral; $\mathrm{V}$ = cuidado à criança; $\mathrm{O}$ = identificar a forma como os pais percebem o cuidado.

\subsection{SEGUNDA ETAPA: LOCALIZAÇÃO E SELEÇÃo DOS ESTUDOS}

Nesta etapa, procedeu-se a definição dos descritores relacionados à temática, utilizando os componentes da estratégia PVO. Dessa forma, os termos selecionados foram: cerebral palsy; parents; child care (inglês); paralisia cerebral; pais; cuidado à criança (português) e parálisis cerebral; padres; cuidado del niño (espanhol). Em seguida os termos foram submetidos aos Descritores em Ciências da Saúde (DECS) para identificação de termos semelhantes. Posteriormente, construíram-se as estratégias de busca submetidas à base de dados. Para tanto, foram aplicados os operadores booleanos aos componentes da escala PVO, respeitando a seguinte composição: (P) AND (V) AND (O).

A pesquisa foi realizada a partir da busca eletrônica e sistemática de artigos indexados na base de dados Psynet APA (American Psychological Association). A escolha pela base se deu devido ao interesse por estudos empíricos realizados na área da psicologia, objetivando o enfoque prioritariamente psicológico acerca dos cuidados na paralisia cerebral. Em relação ao período correspondente da busca não houve data limite de início e o término foi agosto de 2014.

\subsection{TeRCEIRA ETAPA: AVALIAÇÁO CRÍTICA DOS ESTUdOS}

A avaliação crítica dos estudos foi desenvolvida em três fases: 1) refinamento inicial; 2) teste de relevância I; e, 3) teste de relevância II. No refinamento inicial buscou-se excluir artigos repetidos, incompletos e indisponíveis (artigos pertencentes a revistas que não disponibilizavam gratuitamente as pesquisas na íntegra).

O teste de relevância I se deu a partir da análise dos abstracts e selecionou os estudos que obtivessem respostas afirmativas às seguintes questóes: estar em forma de artigo científico; quanto ao delineamento do estudo, pesquisas empíricas (observacionais e experimentais), excluindo-se pesquisas documentais, revisóes sistemáticas e metanalises; dentro dos idiomas estabelecidos; adequado ao objetivo e tema investigado (percepção de pais sobre o cuidado a crianças com paralisia cerebral) e que tivessem como participantes apenas os pais cuidadores de crianças com PC.

O teste de relevância II foi realizado por dois juízes externos que realizaram a leitura dos artigos na íntegra. Este teste avaliou os seguintes aspectos: clareza na investigação do problema, adequação do método aos objetivos e descrição dos resultados em acordo com a metodologia proposta. 


\subsection{QUARTA ETAPA: COLETA DE DADOS NOS ARTIGOS}

Após a aplicação dos testes de relevância, iniciou-se a retirada das informações relevantes aos objetivos da presente revisão. Esta etapa correspondeu à definição das variáveis a serem analisadas e a coleta sistemática das mesmas. Escolheu-se investigar as seguintes categorias de dados: palavras-chave, ano da publicação, autores, local da publicação, idioma, objetivos da pesquisa, forma de abordagem do problema, instrumentos e participantes.

\subsection{QUINTA ETAPA: ANÁLISE E CATEGORIZAÇÃO DOS DADOS}

Os dados extraídos dos estudos foram dispostos em planilhas utilizando o Excel 2010 para melhor organização das informaçóes, gerando assim uma caracterização inicial. Os objetivos dos artigos, foco principal das análises aqui propostas, foram submetidos ao software Nvivo 10 para decodificação por cluster (valor atributo) e aplicação de análise de conteúdo. Desta forma, foram realizadas as seguintes etapas: 1) organização e gerenciamento das fontes de dados no software; 2) codificação das fontes; e, 3) visualização dos resultados de codificação.

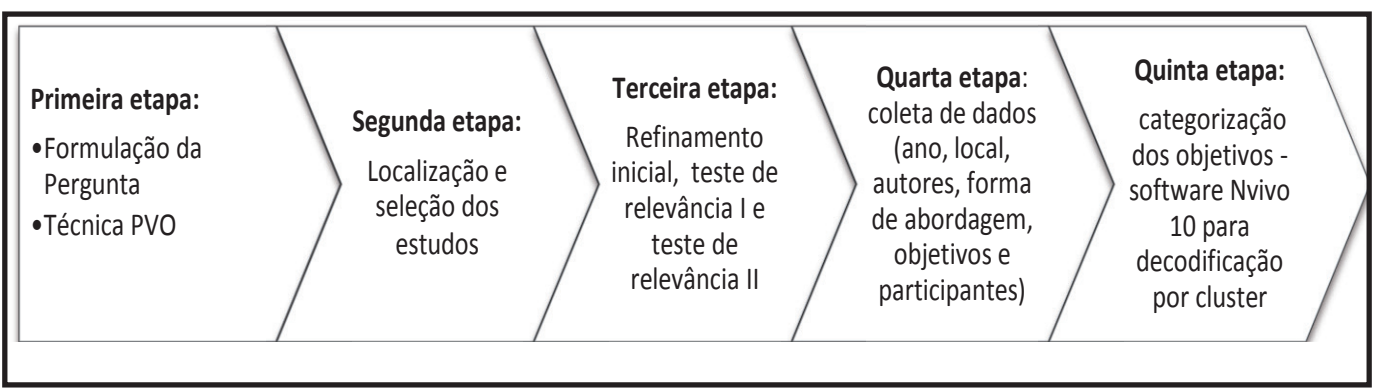

Figura 1 - Fluxograma das etapas de seleção e avaliação dos artigos

Fonte: elaboração própria

\section{Resultados E discussão}

A busca eletrônica resultou em 279 artigos revisados por pares, os quais foram submetidos à avaliação para verificar a possibilidade de inclusão na presente revisão. A avaliação dos artigos se deu por meio de três etapas: a) o refinamento inicial - realizado por apenas um pesquisador para excluir artigos incompletos, repetidos e indisponíveis; b) o teste de relevância I - aplicado por um pesquisador sobre os abstracts dos artigos levantados; c) teste de relevância II - aplicado por dois juízes a partir da leitura dos artigos na íntegra.

No refinamento inicial foram excluídos 41 estudos que se mostraram incompletos, repetidos ou indisponíveis. Sendo assim, 238 artigos foram submetidos ao Teste de relevância 1.

A partir do Teste 1 foram excluídos 214 estudos: 23 por não estarem na forma de artigo científico; dez por não serem empíricos; 48 em que os participantes não eram os pais de crianças com PC; e, em 133, os objetivos não se adequaram ao objetivo da revisão sistemática. Dentre as razóes pelas exclusóes: estudos ligados à medicina e relatos clínicos (46); retratavam terapias motoras (36); validaçôes de escalas motoras (14); estudos epidemiológicos (12); avaliavam as limitaçóes da criança com PC (oito); estudos ligados à área escolar (cinco); avaliaçóes de serviços e programas de reabilitação (quatro); ligados à área da fonoaudiologia (três); apresen- 
tavam a percepção dos profissionais prestadores de serviços (três); ligados à odontologia (um); e por fim, comparava a paralisia cerebral a outras condições - Síndrome de Down (um).

Aos 24 artigos restantes foi aplicado o teste de relevância II. O índice de concordância entre os avaliadores externos foi excelente $(k=0,91)$. O kappa é uma medida de concordância entre observadores e mede o grau de concordância além do que seria esperado tão somente pelo acaso e varia geralmente de 0 -1, em que um grande número significa maior confiabilidade, valores próximos ou menores que zero sugerem que o acordo é atribuível ao acaso. Foram excluídos dois artigos e a amostra final abarcou $22(\mathrm{~N}=22)$ artigos que contemplaram os objetivos da revisão.

Em relação à abordagem utilizada pelas pesquisas que compuseram a amostra final $(\mathrm{N}=22), 12$ se declararam quantitativas e dez qualitativas. Os estudos qualitativos utilizaram entrevistas orientadas por diferentes questionários e diários de campo.

Dentre os instrumentos mais utilizados, destacou-se a GMFM (Sistema de classificação da Função Motora Grossa). Em associação à escala GMFM, foram utilizadas escalas que buscaram avaliar a percepção dos pais sobre o suporte social: Family Support Scale (FSS); Family Support child (FSC); Sarason's Social Support Questionnaire; Social Support Questionnaire; Dunst Social Support Scales; Multidimensional Scale of Perceived Social Support e Interpersonal Support Evaluation List). Outros se mostraram sensíveis às necessidades da família (Family Needs Survey), ao estresse percebido (Parenting Stress Index) e aspectos ligados a atributos pessoais e contextuais: Strengths and Difficulties Questionnaire, Beck Depression Inventory; Child Behavior Checklist; Coping Inventory; Enviroment Scale (FES) e Family Expectations of Child (FEC).

Aos 22 artigos que constituíram a amostra final, realizou-se exploração dos objetivos apresentados a partir da aplicação da análise de conteúdo por clusters utilizando-se o software Nvivo 10. Para tanto, foi realizada a organização das fontes de informação (Etapa 1) e codificação dos objetivos (Etapa 2) por meio da identificação de Nodes ou Nós.

Os nós se apresentam como estruturas com a função de armazenamento de informação codificada e podem assumir diferentes significados. No presente estudo, os "nós" assumiram a função de categorias que identificaram as temáticas exploradas pelos artigos. Por se tratar de uma análise de conteúdo, esses "nós” receberam os códigos (fragmentos dos objetivos), formando categorias de informaçóes.

Após a codificação das fontes, realizou-se uma análise de cluster para visualizar os dados (Etapa 3) por meio da identificação de grupos de Nós. Para tanto foi utilizada a técnica de análise de cluster por valor atribuído, do software NVIVO 10, com coeficiente Jaccard que agrupa elementos por similaridade. Neste caso específico, foram inseridas características categóricas (atributos) que serviram de elementos comuns entre determinados nós, formando clusters (Figura 1). O Coeficiente Jaccard utilizado nesta técnica considera dados binários, em que se entende: 1 = presença; 0 = ausência (Figura 2). 


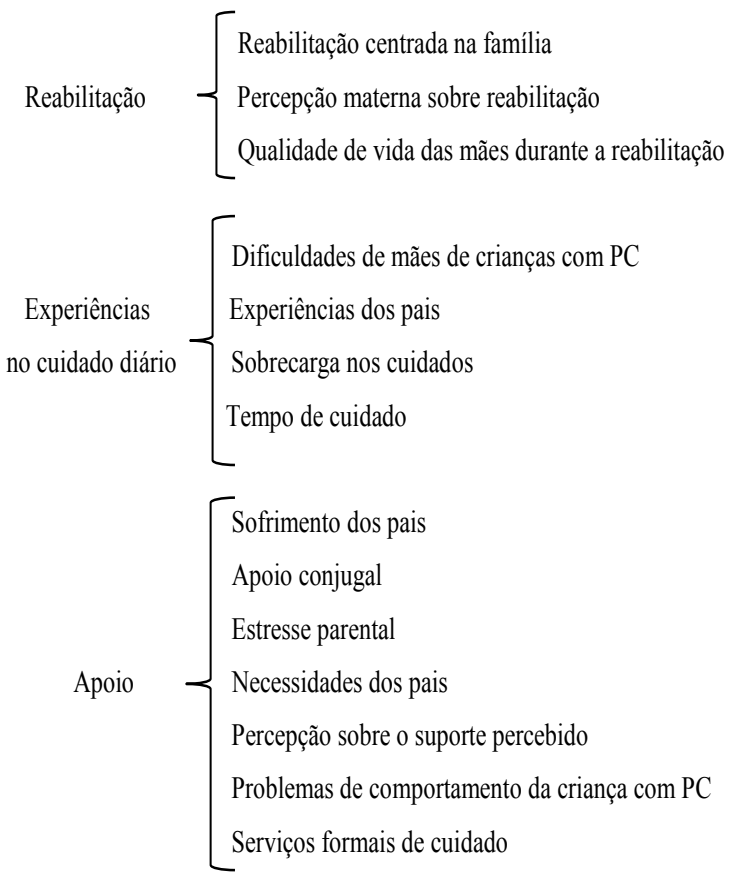

\section{Satisfação parental $\longrightarrow$ Experiências dos pais relacionadas a procedimentos cirúrgicos}

Experiências na alimentação $\left\{\begin{array}{l}\text { Intervenção na prática de alimentação } \\ \text { Dificuldades de mães durante a alimentação } \\ \text { Experiências de mães na alimentação }\end{array}\right.$

Figura 2 - Clusters

Fonte: elaboração própria

A análise de cluster aplicada aos nós dos objetivos resultou em cinco grandes agrupamentos, a saber: 1) reabilitação; 2) experiências no cuidado diário; 3) apoio; 4) satisfação parental; e, 5) experiências na alimentação.

\subsection{Agrupamento - REABILITAÇÁo (DOIS ESTUdOS)}

$\mathrm{O}$ primeiro agrupamento reuniu artigos cujos objetivos mencionavam as seguintes temáticas: reabilitação centrada na família; percepção dos pais e qualidade de vida das mães durante a reabilitaçấo. De maneira geral, os estudos ressaltaram a importância da família durante 
esse processo, no entanto, os objetivos propostos se mostraram diferenciados (GIBSON et al., 2012; PRUDENTE; BARBOSA; PORTO, 2010).

Na pesquisa de Gibson et al. (2012) o objetivo foi descrever as expectativas de 6 pares parentais sobre a reabilitação e os dados apresentados revelaram que as percepçóes estavam associadas à possibilidade do filho vir ou não a caminhar, a julgamentos como ser ou não "bom pai ou boa máe" e ao manter-se esperançoso (a). Já no estudo de Prudente, Barbosa e Porto (2010) o foco principal foi a percepção acerca da qualidade de vida de 100 mães após um período determinado de reabilitação (10 meses). Seus resultados não demonstraram mudanças significativas na saúde dessas mães, concluindo que a qualidade de vida materna não estava relacionada à melhora na função motora da criança após a intervenção.

A análise desta primeira categoria indicou, portanto, duas possibilidades de investigação acerca dos cuidados vistos a partir da reabilitação da criança com PC: ora por meio de atributos como percepçáo e expectativas dos pais, ora a partir dos resultados impactantes de tais açóes. Embora tenham apresentado avanços ao refletir a reabilitação a partir da percepção dos pais, os dados apresentados corresponderam apenas à parte do universo de cuidados, não contemplando, por exemplo, a intensa rotina, aspecto que correspondeu à segunda categoria temática gerada pela análise de cluster.

\subsection{AGRUPAMENTO: EXPERIÊNCIAS NO CUIDADO DIÁRIO (SETE ESTUDOS)}

Essa categoria englobou pesquisas com os seguintes objetivos: relatar as dificuldades de mães de crianças com PC; descrever a experiência dos pais e avaliar a sobrecarga e tempo de cuidado. Os trabalhos direcionados às dificuldades das mães ressaltaram a cultura e as condiçóes sociais precárias que estariam permeando de maneira negativa os cuidados às crianças com PC (KURTUNCU et al., 2013; HUANG; KELLETT; ST JOHN, 2012; HUANG; KELLETT; ST JOHN, 2010).

Kurtuncu et al. (2014) deram voz às mães mulçumanas de 15 crianças com PC moradoras da Turquia e detectou sérias dificuldades que incluíam problemas nas relaçóes pessoais, assim como nas relaçóes conjugais, dificuldades no processo de inclusão social e dificuldades econômicas associadas aos cuidados e educação da criança com PC. Em consonância com estes achados, os trabalhos de Huang, Kellett e St John (2012) e Huang, Kellett e St John (2010) com 15 mães chinesas apontaram que mães de crianças com PC além de permanecerem solitárias no papel de cuidadoras principais, eram hostilizadas pelas famílias de seus maridos, tendo, portanto, pouco ou nenhum apoio nos cuidados aos seus filhos.

As dificuldades dos pais foram relacionadas às experiências de cuidados diários nos estudos de Alaee et al. (2014) e Weiss, Marvin e Pianta (2007). A pesquisa de Alaee et al. (2014) foi realizada no Irã e trouxe o relato de 20 pais acerca dos serviços oferecidos, apontados como inadequados, com interaçóes não suportivas, limitaçóes sociais e isolamento social. A partir das entrevistas, os pesquisadores concluíram que cuidar de uma criança com PC expunha os pais a dois tipos de desafios diferenciados: ora relacionados às características da criança, a partir de percepçóes acerca das limitaçóes e adoecimento constante; ora devido à falta de instalaçóes e atenção a suas necessidades, destacando a falta de apoio formal. 
Weiss, Marvin e Pianta (2007) buscaram descrever e classificar padrões e estratégias utilizadas por 40 famílias americanas no cuidado ao filho com comprometimento motor de moderado a grave. A pesquisa apontou quatro grupos com características diferenciadas. No grupo 1, intitulado Traditional, as mães exerciam a maior parte dos cuidados às crianças com $\mathrm{PC}$, não trabalhavam fora e os pais contribuíam menos nas tarefas de cuidado. $\mathrm{O}$ grupo 2 , Parenting Team, era composto por duas famílias monoparentais trabalhando juntas para atender as necessidades da criança com PC, todos participando dos cuidados. O grupo 3, Extended Family Involvement, abarcou famílias que dependiam fortemente de sua família estendida e por fim, o grupo 4, Formal Support, envolveu famílias que dependiam basicamente do apoio formal de creches, pré-escolas ou outros serviços profissionais.

Os resultados demonstraram que as diferentes estratégias encontradas pelas famílias ao realizarem os cuidados se mostraram, de maneira geral, bem sucedidas. No entanto, a avaliação quanto ao nível de adaptação e o sucesso encontrado nas estratégias adotadas apontaram que o grupo de famílias classificadas como Extended Family Involvement se mostrou o mais bem sucedido, associado com os níveis mais baixos de estresse. Já o grupo Formal Support se mostrou como o menos sucedido, na medida em que esteve associado com níveis maiores de estresse e maior número de hospitalizaçóes. Os demais grupos: Traditional e Parenting Team demonstraram índices intermediários, mais próximos, no entanto, dos padróes encontrados no grupo Extended Family Involvement (WEISS; MARVIN; PIANTA, 2007).

Além das dificuldades sociais relatadas e dos padrões de cuidados frente às diferentes conjunturas familiares e de rede de suporte, a sobrecarga se mostrou como um tema relevante na análise de cluster. Nesse sentido, a pesquisa de Davis et al. (2010) explorou a percepção de 24 mães e 13 pais a partir da avaliação do impacto de cuidar de uma criança com PC de maneira longitudinal, da infância à adolescência. Os resultados indicaram que os pais de crianças com maior comprometimento relataram qualidade de vida mais pobre, experimentando maior sofrimento devido a maior sobrecarga.

A quantidade de tempo utilizado pelas mães nos cuidados aos filhos com PC também esteve relacionado à sobrecarga e foi investigado por Sawyer et al. (2011). Os resultados apontaram que em média, as mães passavam 6,0 horas de 24 horas nos dias de semana e 8,3 horas de 24 horas nos finais de semana cuidando de seus filhos com PC, sendo que quanto maior a quantidade de tempo envolvida, maiores foram os índices de adoecimento mental materno associado a distúrbios depressivos.

Tais resultados destacaram a necessidade de apoios, principalmente às mães, como formas de reduzir o impacto real da prestação desses cuidados, incluindo, nesse sentido, fontes capazes de gerar descanso, assim como suporte na prevenção e tratamento adequado aos problemas de saúde mental. Aspecto ressaltado no terceiro agrupamento gerado pela análise de cluster: o apoio.

\subsection{Agrupamento: apoio (oito eStudos)}

Esta categoria é constituída pelo maior número de variáveis temáticas, a saber: sofrimento dos pais (AL-GAMAL; LONGO, 2013), apoio conjugal (BUTTON; PIANTA; MARVIN, 2001), estresse parental (BUTTON; PIANTA; MARVIN,, 2001), necessidades dos pais (ALMASRI et al., 2012; BAMFORD; GRIFFITHS; KERNOHAN,1997), percepção sobre o 
suporte recebido (PFEIFER et al., 2014; FISS et al., 2014), problemas de comportamento da criança com PC (SIPAL et al., 2010) e serviços formais de cuidado (DAMIANI et al., 2004).

Em relação à variável sofrimento dos pais, a pesquisa de Al-Gamal e Longo (2013) que teve como objetivo avaliar o grau de sofrimento de 204 pais de crianças com PC moradores da Jordânia indicou que mais de $60 \%$ se sentiam, em algum momento, nervosos e estressados. Concluíram que a deficiência grave na criança estava associada ao alto sofrimento psíquico dos pais e ao baixo apoio de amigos.

Além do sofrimento, as variáveis: apoio conjugal e estresse parental mostraram-se relevantes na análise do apoio percebido pelos pais e fizeram parte dos objetivos da pesquisa de Button, Pianta e Marvin (2001) com 64 famílias americanas. Os resultados apontaram que as mães relatavam maior necessidade de assistência prática dos parceiros e não apenas de suporte emocional. Além disso, o maior apoio diário dos parceiros se mostrou como preditor significativo do estresse vivenciado, sendo que quanto maior era o apoio, menor era o estresse percebido pelas mães.

O tema relacionado às necessidades dos pais se mostrou o foco principal do estudo de Almasri et al. (2012) realizado com 579 pais (80\% mães) participantes de programas de reabilitação em hospitais nos EUA, Canadá e México. O objetivo foi avaliar os perfis de necessidades relacionados à criança, família e serviços oferecidos, sendo utilizados questionários para acessar tais informaçóes. Os resultados demonstraram que apesar da heterogeneidade entre os indivíduos com PC e suas famílias, os perfis puderam ser classificados em baixo ou alto grau de necessidades.

Desse modo, nas famílias onde o grau de necessidades se mostrou baixo, os pais demonstraram gerenciar de forma eficaz as condições de saúde de seus filhos, com relaçóes familiares fortes, maiores rendas e possibilidades da criança vir a caminhar. Já nas famílias com maiores restriçóes quanto à mobilidade da criança e menores rendas relatadas, as necessidades por suporte social se mostraram maiores, principalmente financeiro, devido ao impacto advindo com a paralisia cerebral.

De maneira similar, o estudo de Bamford, Griffiths e Kernohan (1997) com 12 famílias irlandesas teve por objetivo investigar as necessidades dos pais e o grau de satisfação acerca da prestação de serviços oferecidos. Os resultados foram categorizados de acordo com a qualidade da ajuda prestada pelos profissionais (médicos, terapeutas, assistentes sociais e enfermeiras) e pelo tipo de acesso e ajuda recebida pelos familiares nas seguintes situaçóes: crise, aconselhamento profissional, serviço de cuidado pago, cuidados domiciliares (residential care), orientação educacional, explicaçóes médicas e suporte familiar/amigos.

Os resultados de Bamford, Griffiths e Kernohan (1997) indicaram que as piores avaliaçóes acerca das necessidades atendidas pelos pais referiram-se aos serviços prestados principalmente pelos médicos e assistentes sociais, com exceção dos cirurgióes ortopédicos, indicando pouca sensibilidade desses profissionais nos momentos de crise e frente à necessidade de aconselhamento. Além disso, as famílias se sentiam sós ou com ajuda apenas dos familiares e poucos amigos. 
Tendo em vista as constatações preocupantes quanto à falta de apoios formais existentes aos pais, alguns estudos, como os de Fiss et al.(2014) e Pfeifer et al. (2014) investigaram os benefícios acerca de outras fontes de apoio aos pais cuidadores. Para Pfeifer et al. (2014) o apoio dos membros da família e amigos foram considerados altamente benéficos à saúde de 50 famílias brasileiras, apesar da carência em relação aos serviços formais. Em perspectiva similar, Fiss et al.(2014), ao investigar 398 famílias americanas, indicaram que o adequado funcionamento familiar esteve relacionado ao bom suporte social que envolvia a família e os amigos.

$\mathrm{O}$ apoio se mostrou, portanto, como fundamental aos pais, principalmente quando havia problemas de comportamento infantil associado (SIPAL et al., 2010). Entretanto, nem sempre as famílias com maiores necessidades de apoio encontraram o devido suporte. Foi o que ressaltou a pesquisa de Sipal et al. (2010) ao investigar 110 pais holandeses de crianças com PC e constatar que o relato daqueles cujos filhos apresentavam problemas de comportamento, apontavam para a falta de relaçôes sociais favoráveis devido às dificuldades de locomoção e comunicação do filho, relacionadas, portanto, ao agravamento dos problemas de comportamento do mesmo.

Por fim, o apoio se mostrou associado aos cuidados formais, objetivo da pesquisa realizada por Damiani et al. (2004) que avaliou a utilização de serviços de folga temporária e descanso planejado por 468 pais canadenses. A pesquisa buscou retratar o quanto os pais dispunham de informaçóes acerca desse serviço, sobre sua utilização, implicaçóes financeiras e barreiras encontradas.

Os participantes eram, em sua maioria, mulheres (94\%) e demonstraram preocupaçóes quanto à carência no oferecimento desse tipo de serviço específico. Nesse sentido, 46\% da amostra já havia utilizado esse serviço, assumindo de maneira solitária os custos envolvidos (40\%). Em 52\% das vezes utilizadas, os serviços eram realizados por um profissional treinado, em $44 \%$ das ocasióes, realizados por babás, amigos e estudantes e em $4 \%$ algum parente. Quanto à frequência no uso e razóes declaradas para sua utilização, 73\% relatou ter utilizado esse serviço mais de seis vezes, desses, $40 \%$ declarou ter utilizado devido a férias e folgas planejadas, 19\% devido à ajuda nas tarefas diárias e por fim, a socialização da criança (10\%).

O apoio aos pais envolveu desse modo, o alívio ao sofrimento e estresse, além de atuar na satisfação das necessidades práticas do dia-a-dia a partir da rede de amigos, famílias e serviços formais de cuidado. A disposição desses elementos constituiu parte do universo dos cuidados, a outra parte correspondeu aos serviços médicos e terapêuticos avaliados a partir da satisfação dos pais gerando o quarto agrupamento: satisfação parental.

\subsection{AgrupAMENTO - SATISFAÇÃo PARENTAL (TRÊS ESTUDOS)}

As pesquisas relacionadas à satisfação parental estiveram associadas principalmente às práticas FCS (Family-centred service) focadas nas famílias e pautadas em parcerias colaborativas entre família e profissionais (WANG; PETRINI; GUAN, 2014; MORGAN; TAN, 2011) e à experiência dos pais durante os procedimentos cirúrgicos sofridos pelos filhos.

Em relação às práticas FCS, a satisfação parental se mostrou baixa no estudo de Wang, Petrini e Guan (2014) destacando o quanto os pais almejavam acesso a informaçóes 
sobre a deficiência, referência de serviços, sensibilidade dos prestadores de serviços e ambiente caloroso. Experiências positivas, no entanto, foram relatadas no estudo de Morgan e Tan (2011), realizado no Camboja, com moradores de regióes rurais e demonstrou que as práticas focadas nas famílias foram pautadas em parcerias com os profissionais do centro de reabilitação em que fora realizada a pesquisa.

No estudo de Morgan e Tan (2011), os pais de crianças com menor comprometimento percebiam os profissionais como "especialistas" e os pais de crianças com comprometimentos mais severos eram mais propensos a relatar sentimentos negativos em relação aos profissionais, valorizando menos a colaboração. Tais resultados evidenciaram a baixa satisfação relatada pelos pais mesmo nas açóes tidas como mais colaborativas.

As dificuldades percebidas se apresentaram com maior severidade no caso das internaçóes e procedimentos cirúrgicos, comuns na paralisia cerebral. Nesse sentido, Iversen, Graue e Clare (2009) entrevistaram pais de nove crianças com PC durante e após a recuperação, indicando o quanto se sentiam vulneráveis e indefesos em uma situação na qual as crianças não podiam expressar seus medos e preocupaçóes.

Além disso, os pais sentiam medo das consequências da cirurgia, além de se mostrarem pouco familiarizados com o que ocorria no hospital. O cansaço extremo também se mostrou marcante ao permanecerem constantemente disponíveis às necessidades das crianças e obrigando a si próprios a serem fortes durante a recuperação.

De maneira geral, os estudos relacionados à satisfação parental destacaram a percepção acerca dos profissionais de saúde. Tais avaliaçóes se mostraram negativas em grande parte, especialmente em relação àqueles com pouca experiência com crianças com PC. Evidenciando o paradoxo existente nas redes e serviços dispostos aos pais, uma vez que quanto maior se mostrou a necessidade relatada por suporte e informaçóes, pior se apresentou a avaliaçáo acerca da satisfação parental quanto aos serviços oferecidos.

Em meio a tantas dificuldades vivenciadas pelos pais, algumas pesquisas objetivaram compreender o cuidado parental a partir da prática de alimentar o filho. E ao delimitarem essa prática, foi possível aprofundar as análises e ressaltar elementos acerca dos apoios, satisfação e reabilitação importantes ao seu desempenho. A alimentação constituiu uma prática desafiadora, dada sua importância à sobrevivência da criança, gerando, portanto, o quinto e último agrupamento: experiência na alimentação.

\subsection{AgRUPAMENTO - EXPERIÊNCIA NA ALIMENTAÇÃo (DOIS ESTUDOS)}

A maioria das crianças com paralisia cerebral têm dificuldades de alimentação, que, se não for gerida, resultam em refeições estressantes, desnutrição crônica, doença respiratória, diminuição da qualidade de vida para o cuidador e criança e morte precoce. Em países com bons recursos, as intervençôes médicas de alto e de baixo custo abrangem desde a alimentação por sonda até treinamento adequado aos pais (ADAMS et al., 2012).

No entanto, em países com alto índice de empobrecimento, tais como Bangladesh, a alimentação por sonda não se mostrava viável e os serviços de treinamento aos pais ocorriam de maneira muito escassa, com eficácia não devidamente avaliada. A partir desse cenário, o 
objetivo do estudo de Adams et al. (2012) foi avaliar a eficácia de uma intervenção de baixa tecnologia para melhorar as práticas de alimentação de 37 cuidadores de crianças com PC de moderada à grave.

Os resultados indicaram que as intervenções realizadas em termos de treinamento dos pais trouxeram melhorias significativas na saúde respiratória das crianças e a cooperação durante as refeiçôes, assim como reduções drásticas no estresse do cuidador. Demonstrando o quanto as açóes concretas de cuidado poderiam ser beneficiadas por intervençóes especializadas, pautadas no apoio e respeito às famílias, sendo que para isso não se mostrava necessário grandes investimentos.

Considerando a importância da alimentação para o desenvolvimento em geral e as dificuldades que crianças com PC apresentam nestas situaçóes especificas, Sleigh (2005) objetivou explorar a experiência de quatro mães inglesas em alimentar suas crianças que apresentavam riscos de desnutrição. Nesses casos, um tubo de alimentação por gastrostomia pode ser oferecido como uma forma de fornecer alimento, uma vez que ignora as principais dificuldades físicas. No entanto, isso não se mostrou como uma decisão fácil. Além dos riscos envolvidos, a escolha pela alimentação gerou preocupaçóes e afetou áreas da vida diária dos pais.

Os momentos de alimentação foram gravados e transcritos. A extração dos conteúdos mais relevantes envolveu a codificação de cada frase que continha significado e as transcrições foram analisadas em dois grupos: a alimentação por via oral e de gastrotomia. Os resultados apresentados refletiram as possíveis escolhas pela via de alimentação mais adequada e o quanto uma boa intervenção poderia vir a contribuir com a melhora da criança com grave deficiência e a diminuição do estresse materno.

Em primeiro lugar, a alimentação oral exigiu atenção individual entre cuidador e criança, o que reforçou a consistência dos cuidados. Além disso, gerou percepçóes de normalidade, aspecto que explicou por que as mães participantes deram à criança uma chance com a alimentação oral.

No caso da alimentação por gastrotomia, os principais pontos de desvantagens relatados foram: perda da normalidade na rotina familiar, experiência hospitalar, gerenciamento em casa do tubo, necessidade de maior assistência profissional em casa, cuidados intensivos, treinamento a outros, complicaçóes médicas e reações de embaraço em público. Em relação às vantagens, observou-se a diminuição do estresse, recuperação mais rápida e ganho de peso.

\section{Conclusāo}

As relaçóes estabelecidas na presente revisão entre os elementos psicossociais investigados destacaram aspectos relevantes na compreensão acerca de como têm se dado o cuidado parental a crianças com PC. Evidenciando, portanto, o processo de reabilitação da criança e a família, assim como as percepçóes parentais acerca do cuidado diário e as dificuldades na execução dos cuidados em diferentes contextos culturais. Além desses, outros aspectos se mostraram importantes para a análise do cuidado à criança com PC, como o sofrimento dos pais, o impacto na qualidade de vida das máes, as principais necessidades quanto a apoios e serviços e a satisfação em relação aos apoios recebidos. 
A maneira como tais elementos foram apontados pelas pesquisas demonstrou a possibilidade de diferentes formas na abordagem do fenômeno, em que foram consideradas, portanto, características sociais e econômicas diversificadas, detectando, desse modo, carências em torno dos serviços de suporte e ajuda aos pais em diversos contextos. Nesse sentido, de maneira geral, a satisfação dos pais em relação ao apoio recebido e aos serviços especializados se mostrou baixa. Em contrapartida, o apoio familiar e de amigos se mostraram benéficos confirmando tendência das pesquisas sobre suporte social (THURSTON et al., 2011; HATZMANN et al., 2009; PATRICK; HAY DEN, 1999).

Frente ao exposto, o cuidado parental na paralisia cerebral pode ser compreendido a partir de adequaçóes que extrapolam os esforços no âmbito familiar, exigindo maior apoio psicossocial e uma rede de suporte capaz de minimizar as adversidades e o risco de adoecimento dos cuidadores. Sendo assim, as análises apontaram que cuidar de uma criança com tais especificidades consistiu em uma tarefa árdua, em parte devido aos riscos e déficits presentes, e em grande parte, devido à falta de serviços especializados sensíveis às necessidades parentais.

Por fim, a revisão destacou tanto a fragilidade em que se encontra a família devido aos custos e limitaçóes sociais impostas, quanto a força e empenho dos pais, principalmente as mães, em cuidarem dos seus filhos, apesar de todas as faltas e dificuldades. Sendo assim, apesar da força do vinculo parental, mostra-se evidente a necessidade de esforços e serviços terapêuticos que auxilie os pais no exercício de seu papel de cuidador. Esta necessidade se justifica, uma vez que para crianças com PC, atividades simples como se alimentar, caminhar, vestir-se constitui um grande desafio que requer o auxilio de seus pais.

Conclui-se que a presente revisão trouxe contribuições significativas ao organizar pesquisas internacionais em torno do tema cuidado à criança com PC, que se apresenta como extremamente importante à Educação Especial e aos serviços de saúde. No entanto, faz-se necessário ressaltar a necessidade da ampliação de novas buscas em outras bases, com novos descritores e estratégias de busca, visando, portanto, o constante mapeamento da área.

\section{REFERÊNCIAS}

ADAMS, M.S. et al. Feeding difficulties in children with cerebral palsy: Low-cost caregiver training in Dhaka, Bangladesh. Child: Care Health and Development, Malden, v.38, n.6, p.878-888, 2012.

ALAEE, N. et al. Psychosocial challenges for parents of children with cerebral palsy: A qualitative study. Journal of Child and Family Studies, New York, v.24, p.2147-2154, 2014.

ALMASRI, N. et al. Profiles of family needs of children and youth with cerebral palsy. Child: Care health and development, Malden, v.38, n.6, p.798-806, 2012.

AL-GAMAL, E.; LONGO, T. Psychological distress and perceived support among Jordanian parents living with a child with cerebral palsy: A cross-sectional study. Scandinavian Journal of Caring Sciences, Malden, v.27, p.624-631, 2013.

ANDRADE DA SILVA, G.; OTTA, E. Revisão sistemática e meta análise de estudos observacionais em Psicologia. Revista Costarricense de Psicología, San Jose, v.33, n.2, p.137-153, 2014.

BAMFORD, D.; GRIFFITHS, H.; KERNOHAN, G. On patient satisfaction in cerebral palsy care. British Journal Social Work, Oxford, v.27, p.605-614, 1997. 
BERGE, J.M.; PATTERSON, J.M.; RUETER, M. Marital satisfactin and mental health of couples with children with chronic health conditions. Families, Systems \& Health, Washington, v.24, n.3, p.267-285, 2006.

BRITNER, P. et al. Stress and Coping: a comparison of self-report measures of functioning in families of young children with cerebral palsy or no medical diagnosis. Journal of Child and Family Studies, New York, v.12, n.3, p.335-348, 2003.

BUTTON, S.; PIANTA, R. C.; MARVIN, R. S. Partner support and maternal stress in families raising young children with cerebral palsy. Journal of Developmental and Physical Disabilities, New York, v.13, n.1, p.61-81, 2001.

CASTRO, A.A. et al. Curso de revisão sistemática e metanálise. São Paulo: LED-DIS/UNIFESP, 2002. Disponível em: <http://www.virtual.epm.br/cursos/metanálise>. Acesso em: 14 abr. 2014 CORDEIRO, A. M. et al. Revisão sistemática: uma revisão narrativa. Revista do Colégio Brasileiro de Cirurgióes, Rio de Janeiro, v.34, n.6, p.428-431, 2007.

CUNNINGHAM, C. Sindrome de Down - uma introdução para pais e cuidadores. 2.ed. Porto Alegre: Artmed, 2008.

CUNNINGHAM, S.D.; WARSCHAUSKY, S.; THOMAS, P.D. Parenting and social functioning of children with and without cerebral palsy. Rehabilitation Psychology, Washington, v.54, n.1, p.109-115, 2009. CURY, V.C.R.; BRANDÁO, M. de B. Reabilitação em paralisia cerebral. Rio de Janeiro: MedBook, 2011. DAMIANI, G. et al. Frequency and determinants of formal respite service use among caregivers of children with cerebral palsy in Ontario. Child: Care, Health \& Development, Malden, v.30, p.77-86, 2004.

DAVIS, E. et al. The impact of caring for a child with cerebral palsy: Quality of life for mothers and fathers. Child: care, health and development, Malden, v.36, n.1, p.63-73, 2010.

FISS, A.L. et al. Family ecology of young children with cerebral palsy. Child: Care Health and Development, Malden, v.40, n.4, p.562-571, 2014.

FLORIAN, V.; FINDLER, L. Mental health and marital adaptation among mothers of children with cerebral palsy. American Orthopsychiatric Association, Washington ,v.71, n.3, p.358-367, 2001.

GERALIS, E. Crianças com paralisia cerebral: guia para pais e educadores. Porto Alegre: Artmed, 2007.

GIBSON, B.E. et al. Children's and parents' beliefs regarding the value of walking: Rehabilitation implications for children with cerebral palsy, Child: care, health and development, Malden, v.38, n.1, p.61-69, 2012.

HATZMANN, J. et al. A predictive model of health related quality of life of parents of chronically ill children: the importance of care-dependency of their child and their support system. Health and Quality of Life Outcomes, London, v.7, p.72-81, 2009.

HORTON, T.V.; WALLANDER, J.L. Hope and social support as resilience factors against psychological distress of mothers who care for children with chronic physical conditions. Rehabilitation Psychology, Washington, v.46, n.4, p.382-399, 2001.

HUANG, Y-P.; KELLETT, U. M.; ST JOHN, W. Cerebral palsy: Experiences of mothers after learning their child's diagnosis. Journal of Advanced Nursing, Malden, v.66, n.6, p.1213-1221, 2010.

HUANG, Y-P.; KELLETT, U.M.; ST JOHN, W. Being concerned: Caregiving for Taiwanese mothers of a child with cerebral palsy. Journal of Clinical Nursing, Malden, v.21, p.189-197, 2012.

IVERSEN, A. S.; GRAUE, M.; CLARE, J. Parents' perspectives of surgery for a child who has cerebral palsy. Journal of Pediatric Health Care, London, v.23, n.3, p.165-172, 2009. 
KURTUNCU, M., et al. Experiences shared through the interviews from fifteen mothers of children with cerebral palsy. Journal Sexuality and Disability, New York, v.33, n.3, p.349-363, 2014.

LIN, S.L. Coping and adaptation in families of children with cerebral palsy. Exceptional Children, Chicago, v.66, n.2, p.201-218, 2000.

LOPES, C.R.M. et al. Carga y dependencia em cuidadores primarios informales de pacientes com parálisis infantil severa . Psicologia y Salud,San Miguel de Salinas, v.22, n.2, p.275-282, 2012.

MORGAN, F; TAN, B.K. Rehabilitation for children with cerebral palsy in rural Cambodia: Parental perceptions of family-centred practices. Child: care, health and development, Malden, v.37, n.2, p.161167, 2011.

PATRICK, J.H.; HAY DEN, J.M. Neuroticism, coping strategies, and negative weil-being among caregivers. Psychology and Aging, Washington, v.14, n.2, p.273-28, 1999.

PFEIFER, L.I. et al. Social support provided to caregivers of children with cerebral palsy. Child: care, health and development, Malden, v.40, n.3, p.363-369, 2014.

PRUDENTE, C.O. M.; BARBOSA, M.A.; PORTO, C.C. Relação entre qualidade de vida de mães de crianças com cerebral paralisia e o funcionamento do motor das crianças, após dez meses de reabilitação. Revista Latino - Americana de Enfermagem, Ribeirão Preto, v.18, n.2, p.3-10, 2010.

SAWYER, M.G. et al. Time demands of caring for children with cerebral palsy: What are the implications for maternal mental health? Developmental Medicine \& Child Neurology, Hoboken, v.53, p.338-343, 2011.

SIPAL, R.F. et al. Course of behavior problems of children with cerebral palsy: The role of parental stress and support. Child: care, health and development, Malden, v.36, n.1, p.74-84, 2010.

SLEIGH, G. Mothers' voice: A qualitative study on feeding children with cerebral palsy. Child: Care, Health \& Development, Malden, v. 31, n. 4, p. 373-383, 2005.

THURSTON, S. et al. System Integration and Its Influence on the quality of life of children with complex needs. International Journal of Pediatrics, Nova York, v.1, p.1-12, 2011.

VIEIRA, M.L. et al. Cuidado e responsividade parentais: uma análise a partir da teoria da história de vida e da teoria do investimento parental. In: OTTA, E.; YAMAMOTO, M.E. (Org.). Psicologia Evolucionista. Rio de Janeiro: Guanabara Koogan, 2009. p.86-95.

WANG, M.; PETRINI, M.A.; GUAN, Q. Evaluation of family-centred services from parents of chinese children with cerebral palsy with the measure of processes of care. Child: care, health and development, Malden, v.41, n.3, p.1-7, 2014.

WEISS, K.L.; MARVIN, R.S.; PIANTA, R.C. Ethnographic detection and description of family strategies for childcare: Applications to the study of cerebral palsy. Journal of Pediatric Psychology, Oxford, v.22, n.2, p.263-278, 1997.

WHITING, M. Impact, meaning and need for help and support: The experience of parents caring for children with disabilities, life-limiting/life-threatening illness or technology dependence, Journal of Child Health Care, London, v.17, n.1, p. 92-108, 2012.

ZHU, B. et al. Strutural equation and log-linear modeling: a comparison of methods in the analysis of a study on caregivers' health. BMC medical Research Methodology, London, v.6, n.49, p.1-14, $2006{ }^{7}$

Recebido em: 13/10/2015

Reformulado em: 16/05/2016

Aprovado em: 30/05/2016 